\title{
Onboard Train Localization with Track Signatures: Towards GNSS Redundancy
}

\author{
Oliver Heirich, Benjamin Siebler \\ German Aerospace Center (DLR), Institute of Communications and Navigation \\ Oberpfaffenhofen, Germany \\ oliver.heirich@dlr.de
}

\section{BIOGRAPHIES}

Oliver Heirich graduates in electrical engineering (Dipl.-Ing.) at the University of Ulm (Germany) in 2008 and started as a development engineer at a sensor manufacturer. Since 2010 he is a research scientist at the Institute of Communications and Navigation at the German Aerospace Center (DLR) in Oberpfaffenhofen. His current research activity involves train localization based on multi-sensor fusion as well as railway track mapping with simultaneous localization and mapping (SLAM) methods.

Benjamin Siebler received his master degree in electrical engineering from the Karlsruhe Institute of Technology (Germany) in 2014. Since 2014 he is a research scientist at the Institute for Communications and Navigation at the German Aerospace Center (DLR) in Oberpfaffenhofen. His current research activity involves train localization with train-side mounted sensors.

\begin{abstract}
Future railway applications, such as train-side collision avoidance, virtual coupling or autonomous train driving demand reliable and accurate train localization. We focus on exclusive onboard train localization without additional way-side infrastructure. Common approaches for onboard train localization are based on measurements of a global satellite navigation system (GNSS). Well-known methods for improved reliability and accuracy are the combination with inertial navigation systems (INS) or dead reckoning. However, these approaches show an unbounded growth of an error for longer GNSS outages. Reliable train localization requires a long-term stability with bounded errors in long tunnels, at underground stations or in long stretches of dense forests.

This paper presents two methods as well as a new system concept for onboard train localization with GNSS redundancy and long-term stability. The first method is signature based train localization approach from independent IMU and magnetometer measurements. The second method introduces an independent speed estimation approach without a wheel speed sensor nor a GNSS.

A track signature is considered here as a signal of a track feature which is sampled over the 1-D positions of a track. It is possible to estimate a location from a comparison of the latest measured signatures and reference signatures from a map. This paper contains further an analysis of the signatures on straight tracks, parallel tracks, a switch, and a curve. The used signatures comprise eight signatures from magnetic, curvatures, attitude and vibration. All signatures use recorded data from real train runs of a regional train.
\end{abstract}

\section{INTRODUCTION}

Location based railway applications focus on increased safety and efficiency. Application examples are known as general train control, moving block operation and automated train operation (ATO). Advanced applications include a train-side collision avoidance system, which exchanges train locations between trains as well as the virtual coupling method for consecutive running trains below the braking distance. One important part of these applications is reliable and continuous train localization with track-selective accuracy. Onboard train localization estimates a location with train-side mounted sensors. In contrast to infrastructure based localization, the onboard train localization uses a map of the railway tracks and does not require additional way-side infrastructure.

In the recent years, there are many onboard train localization approaches with global satellite navigation system (GNSS) as listed in a survey from Marais et al. [1]. However, a solely GNSS based localization method cannot always provide the required continuity and accuracy in the railway environment, especially in tunnels or at degraded signal reception conditions.

A train localization system for safety-critical applications requires redundancies. System redundancy can be achieved with well-known methodologies, such as multiple systems of the same type or hardware redundancy with back-up power supplies, different cables and components. A software redundancy is achieved with different specifications, different programming 
teams, different compilers and programming languages. In contrast, the GNSS redundancy in this paper aims at the train location with independent sensors and methods.

This paper presents a new system concept for onboard train localization and speed estimation with independency of GNSS and wheel speed data and long-term stability. Current train signaling equipment uses wheel speed sensors. The additional independency of wheel speed data is of interest for a simplified retrofit of trains with navigation equipment. Furthermore, the speed measurements are distorted by wheel slip.

The challenges of GNSS independent train localization are an absolute positioning on tracks as well as an independent speed and traveled distance estimation. The aim is rather a gain in reliability than a replacement of GNSS. GNSS data and wheel speed data are still considered as valuable localization information and intended in a combined concept with a complementary multi-sensor fusion. Nevertheless, the proposed method is suitable to operate in GNSS degraded and denied areas, for example in urban canyons, forests and tunnels.

The first method is a signature based train localization approach based on measurements from MEMS (micro-electromechanical system) IMU and a solid-state magnetometer. This localization method uses known track signatures and compares it to the latest measured signature. The second method introduces an independent speed estimation approach without a wheel speed sensor. Both methods have been initially implemented and analyzed in a supervised work by the authors [2]. The proposed methods are suitable to estimate a long-term stable train location without GNSS and wheel speed data.

\section{ONBOARD TRAIN LOCALIZATION METHODS BASED ON GNSS}

The onboard train localization estimates the topological location which consists of a railway track identifier $(i d)$ and a 1-D position $(s)$ on that track. The map links the topological location samples with track feature values that can be measured by onboard sensors. In case of GNSS based train localization, the map contains geo position samples linked with the topological positions for each track.

A simple method for GNSS based train localization is map-matching. There, the nearest topological position is computed from a GNSS position measurement and the map. A simple map-matching algorithm aims on minimizing the distance between the measured position and a track, which is a function of sample points from the map. More advanced methods use estimation filters with motion models and train speed measurements as well as probabilistic measurement models. A well-known method is dead-reckoning on the map from train speed measurements. Speed measurements are often achieved by a wheel speed sensors. However, a railway wheel may slip in acceleration phases or slide in deceleration phases. Position measurements can observe and correct accumulated distance error from the speed integration. Another common approach is the combination of GNSS with an INS (inertial navigation system) for enhanced accuracy and continuity for limited periods of GNSS outages. The INS estimates position, attitude and velocity from IMU (inertial measurement unit) measurements and an INS/GNSS corrects the accumulated errors of the INS with GNSS measurements. Both methods, the dead-reckoning and the INS, are considered as short-term stable estimates with an unbounded error. The error stability of an INS depends mainly on the IMU sensor quality, in particular on the gyroscopes (see [3]).

\section{TRACK SIGNATURES}

A signature is considered here as a signal of a track feature which is sampled over the 1-D position of a railway track. There are two important properties for a suitable track signature: a location dependency with an adequate repeatability on the same locations as well as a separability between signatures of different locations. A signature match of a measured and a recorded signature can observe the along-track position as well as discriminate the different parallel tracks and competitive switch ways. Prior measurement analysis identified appropriate signatures of curvatures, magnetic signals [4], and vibrational signals from train recordings of a regional passenger train at regular service. In the following, the individual signatures are presented over $800 \mathrm{~m}$ of the same six train runs. The train approaches the station "Friedberg" for three times at platform 1 and for three times at platform 2. Therefore, the train passes several scenarios: a straight track, a curve, and a switch followed by straight parallel tracks towards the platforms. The switch is passed facing with three runs to the left switch way and three runs to the right switch way.

\section{Attitude Signatures}

The attitude of a track consists of three angles: bank, slope and yaw angle and the signatures are shown in Figure 1. These attitudes have been computed with an INS/GNSS and are not independent of a GNSS. Nevertheless, we present a signature based INS aiding without GNSS in [5]. These attitude signatures show low frequencies over the track position because the attitude changes are limited for comfort and safety reasons. The curve has a superelevation, in order to compensate centrifugal acceleration during a curve passage. This centrifugal acceleration is limited for safety-reasons, and a superelevation allows higher speeds in curves. This superelevation is represented by the bank angle in the curve scenario. The yaw angle changes 
clearly in the curve. It can be seen, that the bank and yaw signature is different for different switch ways. The reason for the different bank angles in the switch scenario is due to the inclination of the train cabin. The IMU measures a leaning and shaking train cabin caused from curve changes at the switch. The slope angles are very little with $0.5^{\circ}$ when the train runs uphill towards the station and the parallel tracks are leveled at the station.

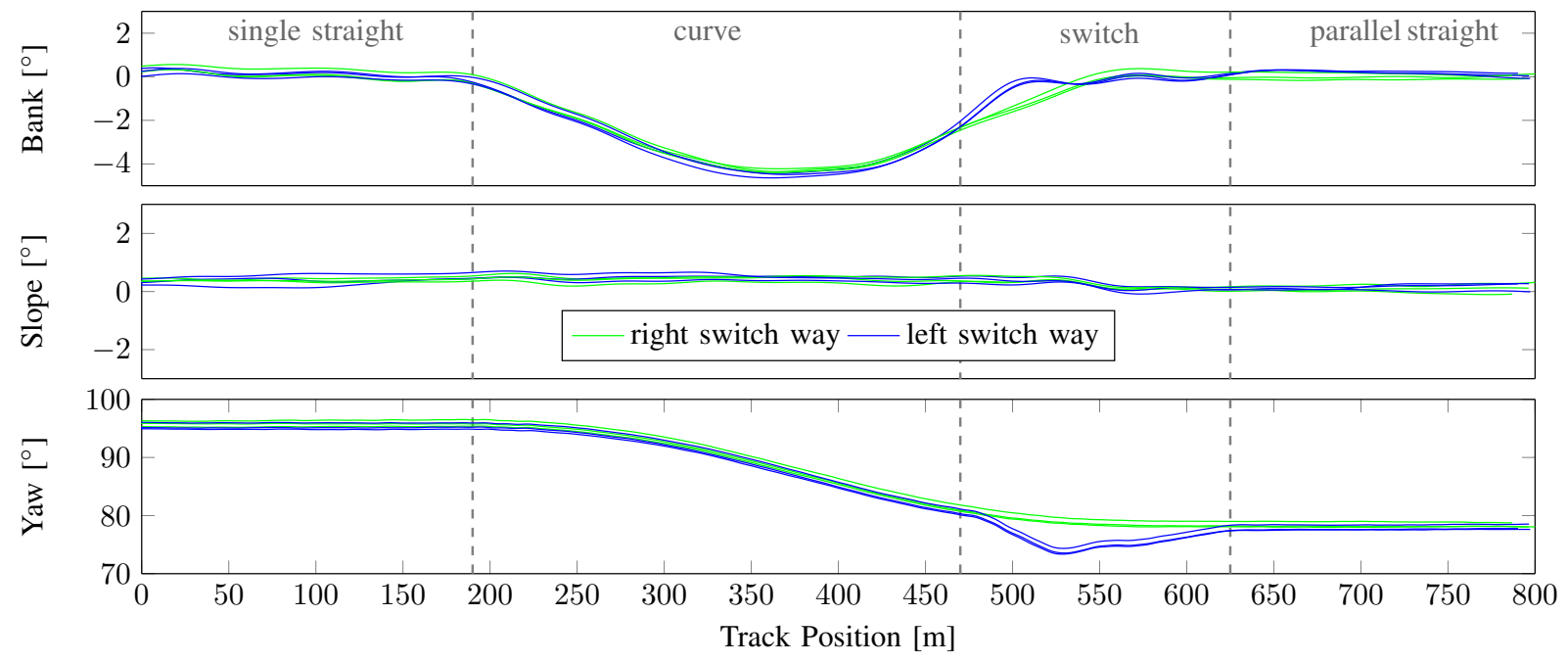

Fig. 1: Attitude signatures of bank, slope and yaw of six train runs.

\section{Curvature Signatures}

A railway track geometry can be characterized by three curvatures: the bank, slope and yaw curvature. A curvature is the reciprocal value of the radius and is defined by an angular change of the bank, slope or yaw angle per distance increment. The use of a curvature instead of radius has practical reasons: A straight track or almost straight track has a curvature of zero or near zero whereas a radius of a straight track is infinite or very large for almost straight. The curvatures are defined in the map over the 1-D track position s for each track. A curvature can be computed from the turn rate $(\omega)$ measurements of a train mounted IMU and the speed $(\dot{s})$ :

$$
\left(\begin{array}{c}
c^{\phi} \\
c^{\theta} \\
c^{\psi}
\end{array}\right)=\left(\begin{array}{l}
\omega^{x} \\
\omega^{y} \\
\omega^{z}
\end{array}\right) \frac{1}{\dot{s}} .
$$

Figure 2 shows the bank, slope and yaw curvature signatures of the six train runs with the four scenarios. The curvature signatures over the track position show low changing signal properties. Especially in case of straight tracks, the curvatures

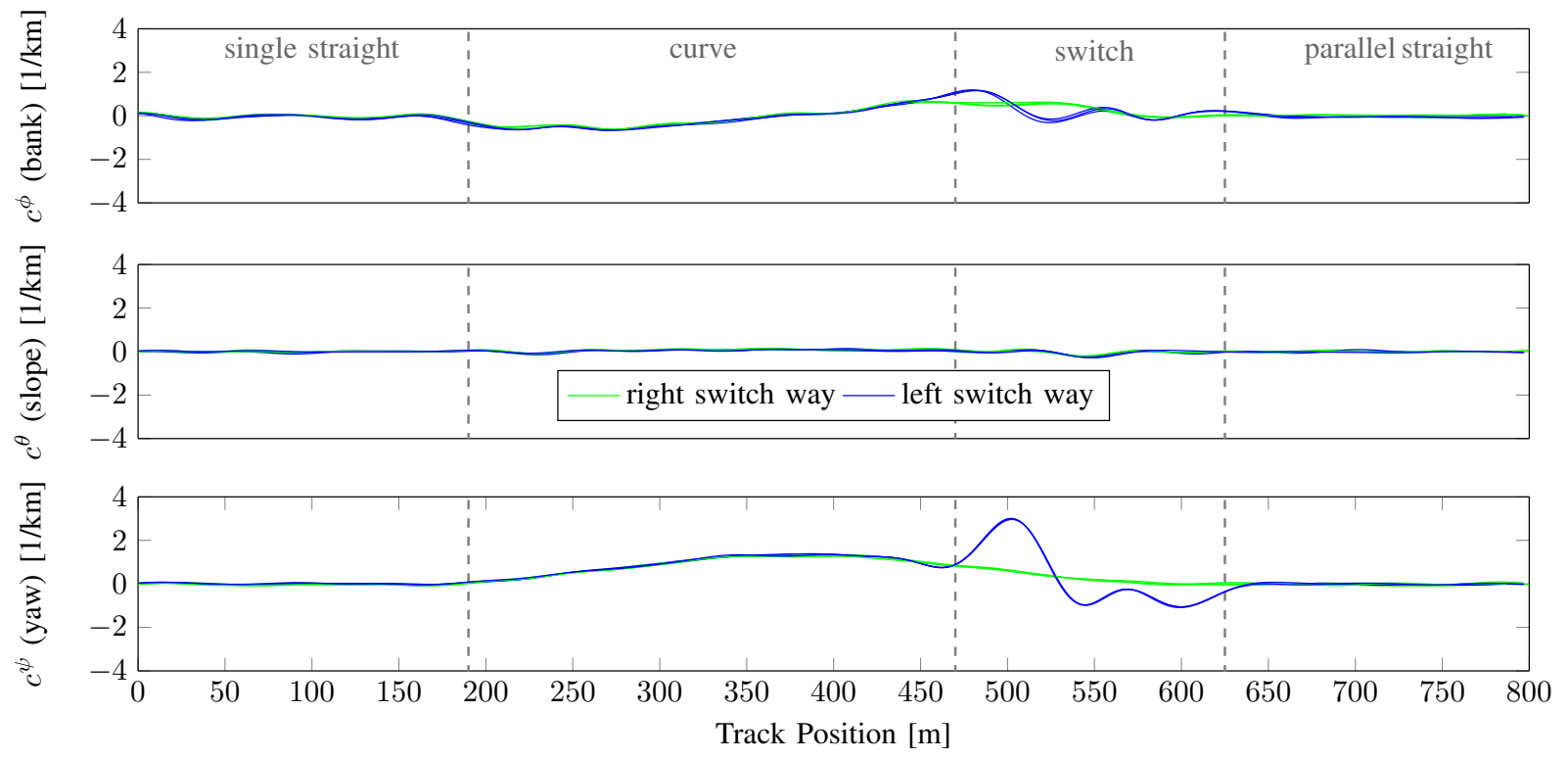

Fig. 2: Curvature signatures of bank, slope and yaw curvature of six train runs. 
are zero and there is no signal for an along-track position update, as seen for the single and parallel straight tracks. The curve can be seen in the yaw curvature and the bank curvature. The bank curvature indicates the roll motion of the train on the transition ramp between the leveled straight track and the superelevation curve. The slope curvature shows the least information in all scenarios. The different switch ways are clearly seen the yaw curvature. The reason for the different bank curvatures in the switch scenario is the same as for the bank angle: The train changes quickly the curves and the train cabin begins to lean and swings back. Finally, the parallel tracks show no difference in curvatures.

\section{Magnetic Signatures}

The magnetic signature is a variation of the earth magnetic field from local ferromagnetic objects. Figure 3 shows six magnetic signatures recorded from a cabin mounted magnetometer. In the straight track and the curved track, the six signatures are very similar, so that there is only the last (blue) signature visible. The switch and the parallel tracks show different signatures for left way or right way, each one with a high repeatability. It can be seen, that there are higher signal frequencies than in the curvature or attitude signatures. This property is beneficial for a continuous along-track positioning. However, other trains and track works can disturb and change the signature [4].

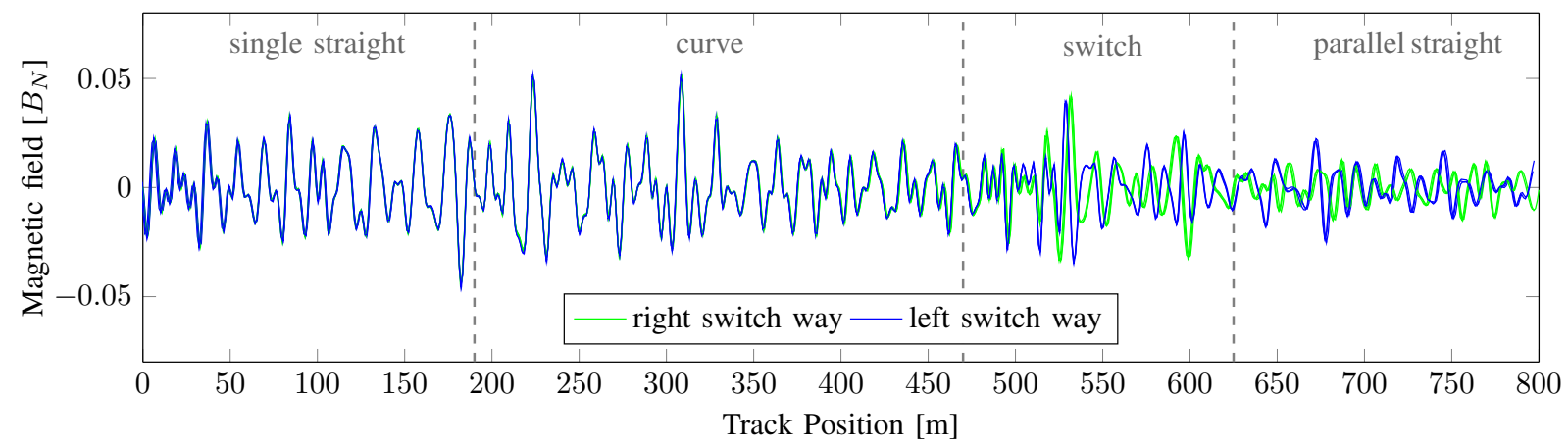

Fig. 3: Magnetic field signatures of six train runs.

\section{VibRATION SignatureS}

Vibrations are oscillatory motions that can be measured with accelerometers and also gyroscopes. Vibrations have many sources and the ones of interest are the exited wheel vibrations for speed measurement and the location dependent vibrations from the tracks. A worse track condition may be favorable for localization. However, track maintenance may also change the signature. Figure 4 shows the vibration signatures of six runs of the vertical acceleration of a bogie mounted IMU. This vibration signature is computed with an envelope that is normalized to the maximum intensity. It can be seen, that the signature has repeatable patterns in the straight and curve section, and separable pattern in the switch area. The parallel straight shows a fade-out of the vibration signature, as the train brakes down get gets slower. The train speed is between $60 \mathrm{~km} / \mathrm{h}$ to $80 \mathrm{~km} / \mathrm{h}$ on the single straight track and the train breaks down to speeds between $60 \mathrm{~km} / \mathrm{h}$ and $40 \mathrm{~km} / \mathrm{h}$ at the parallel tracks.

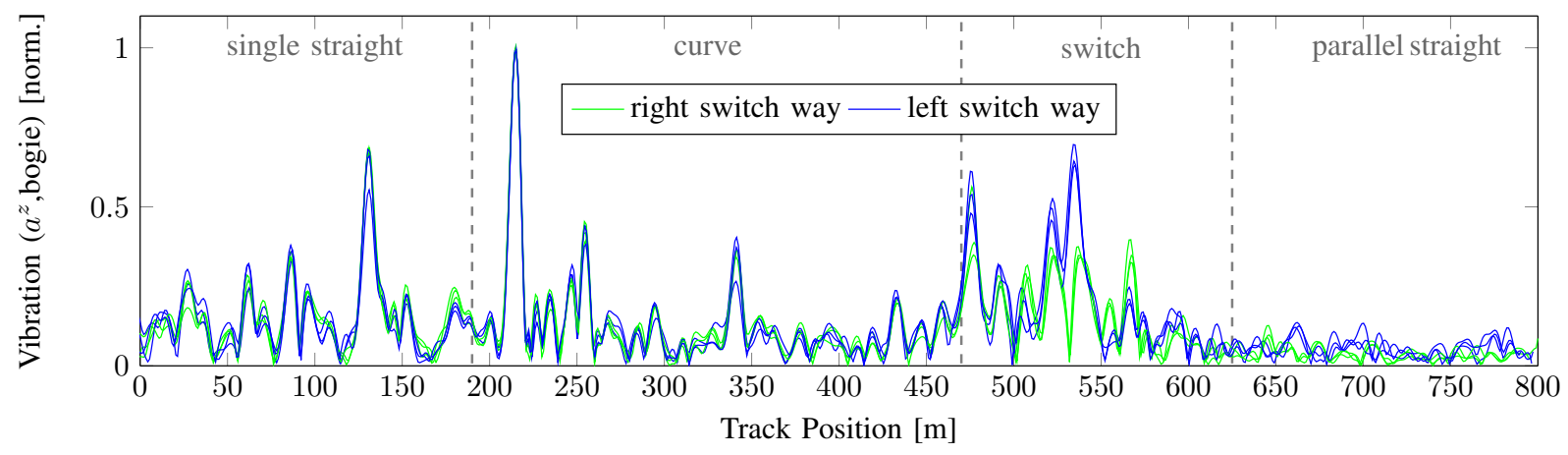

Fig. 4: Vibration signatures of six train runs. 


\section{ONBOARD TRAIN LOCALIZATION METHOD BASED ON TRACK SIGNATURES}

A train localization method with track signatures estimates the location from current measured signatures and reference signatures. These reference signatures are stored in a map for each track. All signatures are sampled with a constant sample distance $(d s)$ and the signature values are stored in combination with the 1-D position.

A signature is created from a measurement sequence of a train-side sensor and train speed information. The measurements are pre-filtered and buffered in combination with speed information. The time depended measurement samples are transformed with a known train speed to location depended samples with a predefined sample distance. This train speed is either measured from wheel-speed sensors, GNSS or INS/GNSS. An alternate train speed estimation method is presented in the next section with IMU and magnetometer measurements. The current signature contains the latest measurements up to a predefined length and is compared within a search window of the reference signatures. The comparison is achieved by signal similarity measures, such as correlation coefficient and the shift position to the highest similarity. This search window is placed at the predicted location and is longer than the actual signature with an additional tolerance length before and after. The current signature is matched within the search window and the result is a maximum similarity score and shift distance to that maximum. Finally, this shift is used for further corrections in an update step of the train location estimator.

In [4], an evaluation of an along-track estimator based on magnetic signature and IMU acceleration measurements has shown errors in the range of the accuracy of a standard GPS-L1 position reference.

\section{TRAIN SPEED ESTIMATION METHOD WITHOUT GNSS AND SPEED SENSOR}

The presented method is a speed and distance estimation without the need of GNSS or wheel speed sensor. The approach uses IMU data, magnetic data and a map. An acceleration measurement in longitudinal train direction (x-axis) is denoted with $z^{\mathrm{a}, \mathrm{x}}$ and contains the train acceleration from traction and brake. This train acceleration can be integrated to the train speed and traveled distance. However, the IMU measures additional accelerations, such as the gravity, vibrations, changing sensor bias and sensor noise. The sensor error model of the longitudinal acceleration measurement is given by:

$$
\underbrace{z^{\mathrm{a}, \mathrm{x}}(t)}_{\begin{array}{c}
\text { acc. meas. } \\
\text { train frame }
\end{array}}=\underbrace{\ddot{s}(t)}_{\begin{array}{c}
\text { train } \\
\text { traction/brake }
\end{array}}+\underbrace{g \cdot \sin (\theta(t))}_{\begin{array}{c}
\text { gravity } \\
\text { portion }
\end{array}}+\underbrace{b^{\mathrm{a}, \mathrm{x}}(t)}_{\begin{array}{c}
\text { bias, } \\
\text { drift }
\end{array}}+\underbrace{n^{\mathrm{a}, \mathrm{x}}(t)}_{\begin{array}{c}
\text { noise } \\
\text { vibrations }
\end{array}} .
$$

Figure 5 shows the integrated train speed from acceleration measurements of a real train run without corrections for gravity and changing bias. It can be seen, that the additional terms of Eq. 2 cause a growing deviation between the GPS speed and the speed estimation based on an integration of acceleration measurements.

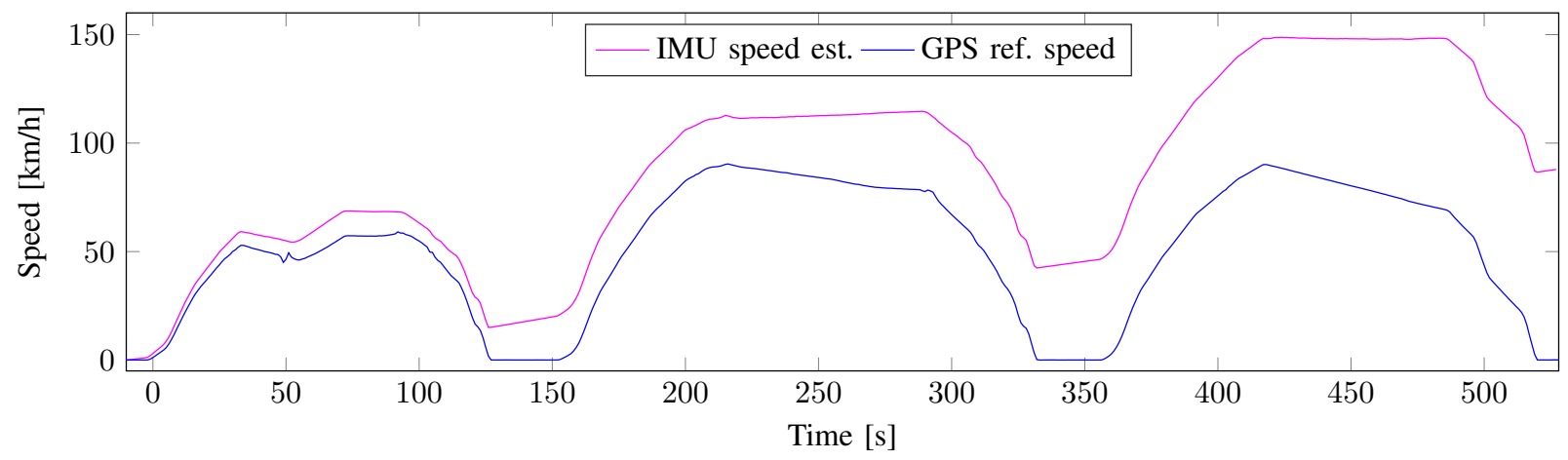

Fig. 5: Train speed from GPS reference and uncorrected integration of acceleration measurements.

In the following approach, the gravity portion is corrected from a slope estimate. This slope is computed from the predicted location estimate and a known slope profile of the map. The corrected acceleration measurement is used as input for the estimation filter. The continuous state-space model for the distance $(d)$, speed $(v)$ and bias $(b)$ estimation is given by the differential equation with the acceleration input $\left(a^{x}\right)$, its measurement noise $\left(n^{a}\right)$ and the prediction noise $\left(n^{b}\right)$ for the bias estimate:

$$
\frac{d}{d t}\left(\begin{array}{l}
d_{t} \\
v_{t} \\
b_{t}
\end{array}\right)=\left(\begin{array}{ccc}
0 & 1 & 0 \\
0 & 0 & 1 \\
0 & 0 & 0
\end{array}\right)\left(\begin{array}{l}
d_{t} \\
v_{t} \\
b_{t}
\end{array}\right)+\left(\begin{array}{l}
0 \\
1 \\
0
\end{array}\right) a_{t}^{x}+\left(\begin{array}{ll}
0 & 1 \\
1 & 0 \\
0 & 1
\end{array}\right)\left(\begin{array}{c}
n_{t}^{a} \\
n_{t}^{b}
\end{array}\right)
$$

A standard Kalman filter estimates the state with the discretized version of the state-space model. The special feature of this approach is the speed measurement updates from the magnetic and vibration measurements. Inertial measurements as well as magnetic signals show repeatable frequency spectrums for the same train speeds and different spectrums at other train speeds 
[6], [7]. In a first phase, inertial and magnetic signals are recorded for each speed within a certain grid (e.g. $0.5 \mathrm{~km} / \mathrm{h})$. The speed is estimated in the second phase via the most likely match of the measured and the recorded speed spectrum. This speed estimation method is prone to heavy outliers, and therefore an outlier detector prevents an update of false speed measurements. Figure 6 shows the train speed from the proposed method, the GPS speed reference as well as accepted or rejected vibration speed estimates. The magnetic speed estimates showed also similar outliers. In this example the independent speed estimation method shows speed errors up to $2.5 \mathrm{~km} / \mathrm{h}$ and an integrated distance error of $52 \mathrm{~m}$ at the end of the $7.6 \mathrm{~km}$ train run. It should be noted, that these first results are from a single run and compared to a GPS reference.

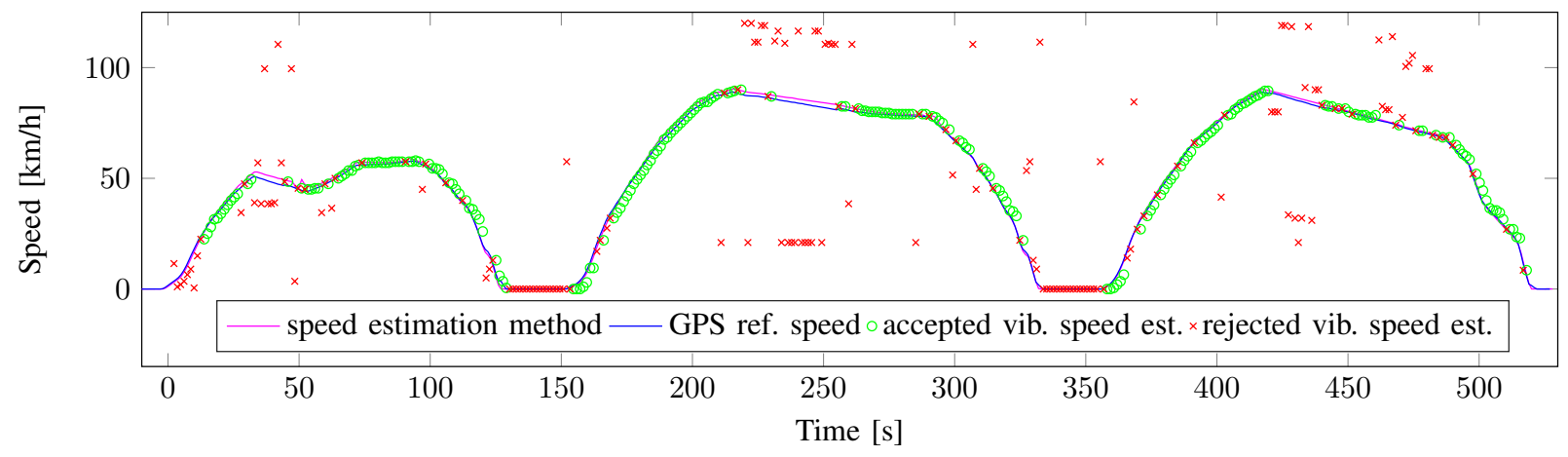

Fig. 6: Train speed: GPS reference and IMU based speed estimation method.

\section{ONBOARD TRAIN LOCALIZATION CONCEPT WITH GNSS REDUNDANCY}

The GNSS and wheel speed sensor independent train localization approach requires at least two methods: first, a localization method from track signatures and second, a speed estimation method. A train localization method from a comparison of map data and measured track signatures has been shown in this paper. Furthermore, the estimation of train speed and traveled distance is vital for the signature processing and location prediction.

Figure 7 shows the block diagram for a complementary train localization concept with track signatures in combination with optional GNSS and wheel speed sensor. The speed $v$ is used as input in several blocks and there are two ways shown for the speed estimate: The INS/GNSS estimates the speed $v^{\prime}$ and in case of no GNSS data, the speed can be estimated from IMU acceleration, vibration and magnetic patterns $\left(v^{\prime \prime}\right)$. A wheel speed sensor is advantageously, but not required. The intended track signatures are the magnetic field signature, the curvature signatures (bank, slope and yaw) and the vibration signatures. There are additional signatures of position and attitude (bank, slope and yaw) of the INS/GNSS. These signatures can be used according to GNSS availability and during short interruptions below bridges and short tunnels. In total, there are five signatures available without GNSS and eight signatures with GNSS availability or for a limited time after GNSS outage.

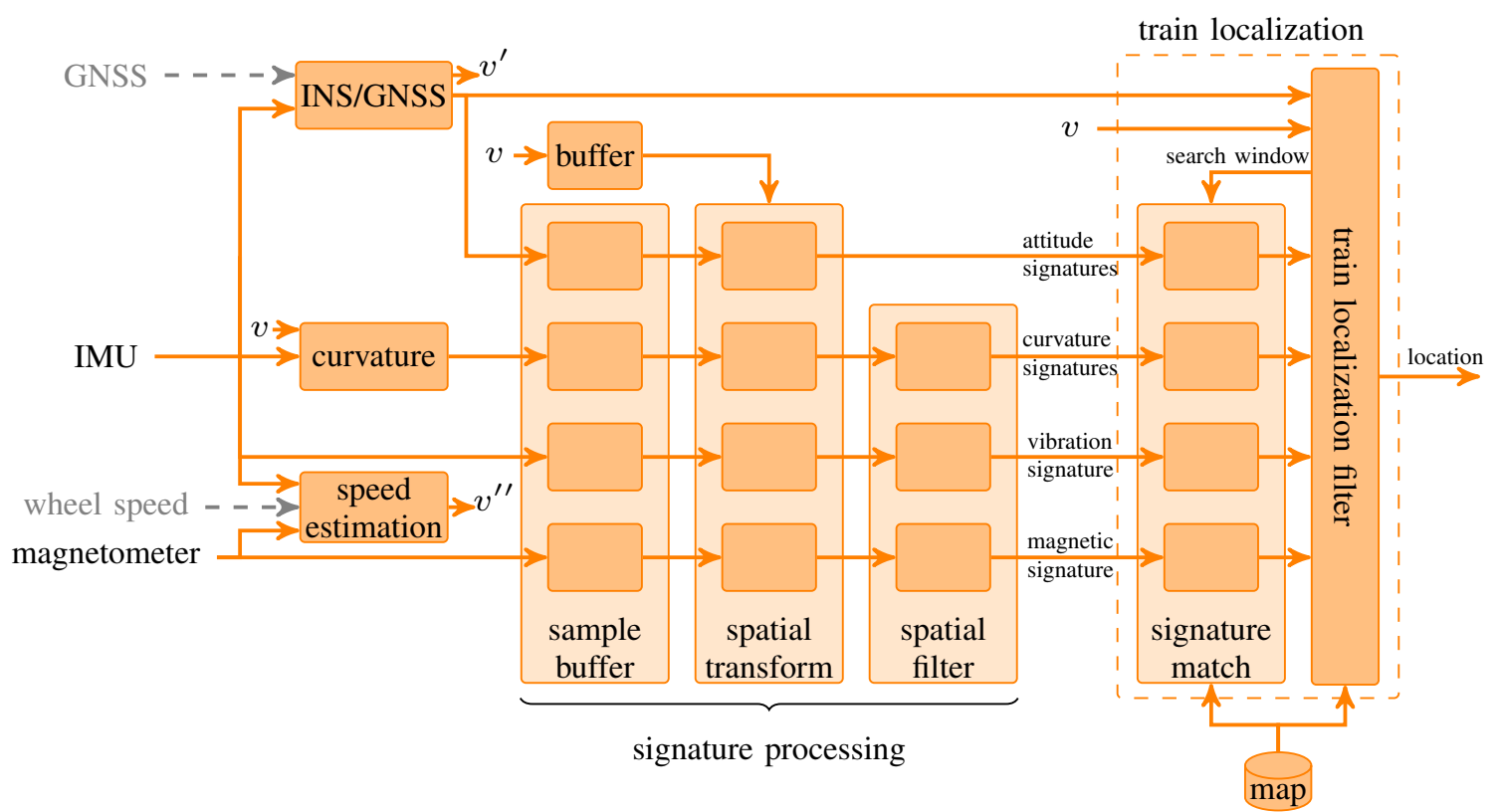

Fig. 7: Onboard train localization concept with track signatures and GNSS redundancy. 
The signature processing blocks are similar for all signatures and consist of a sample buffer, a spatial transformation, and a spatial filter for the current signatures. A signature match block compares the current signatures with reference signatures from the map. The comparison of the signatures is processed within a search window with a larger size than the current signature length. The signature match block reports a similarity measure and a shift distance to the train localization filter. Finally, the train localization filter estimates a train location from all observations.

\section{DISCUSSIONS AND CHALLENGES}

The individual signatures have shown different characteristics: The magnetic signature is the most versatile and best suited for along-track estimation, switch way detection and parallel track identification. However, other trains and track works can disturb and change this signature. Curvature and attitude signatures showed suitable switch way discrimination, but limited capabilities for a continues along-track estimation, with exceptions at curve changes. The vibrations may be used for alongtrack estimation, but showed a speed dependency of the signature. The location dependency of some signatures is not exclusive: as shown with the bank angle and curvature, a curve causes the train cabin to swing and generates an own signature at similar train speeds of the different runs. In conclusion, some of the signatures depend on the sensor mounting, train speed and possibly on the train type. As a consequence, individual signatures demand for individual maps for each setup and train type. Therefore, an automated creation and maintenance of a map is needed, such as a SLAM (simultaneous localization and mapping) method for railway tracks [8].

The presented signature localization method shows already good functionality whereas the speed estimation method is more a proof-of-concept with potential for improvements. The proposed train localization concept can be extended, as there are many different localization methods possible, other sensors, different sensor combinations and estimation filters. The development of new localization methods and the evaluation with real train data is an ongoing task. An advanced train localization system with active redundancy requires a further evaluation unit with a voting logic for the best train location result.

A challenge is the change of a signature over time. In [4], a magnetic signature with a repeatable pattern changed to a different repeatable pattern between two runs separated by two days. Potentially due to track works, the change happened in a relative small section of several hundred meters. Nevertheless, this is a problem for the magnetic localization method. The map has to be maintained and the signatures need to be updated once there is a change detected.

\section{CONCLUDING REMARKS}

The paper presented two methods an d a concept for onboard train localization with and without GNSS and a wheel speed sensors. First, an independent train speed estimation method from IMU and magnetic data has been presented with speed observations from typical signals at certain speeds. Finally, a concept for a complementary multi-sensor train localization method with track signatures, GNSS and a wheel speed sensors is given.

The GNSS independency is achieved by track signatures of curvature, vibration and magnetic field. The individual track signatures showed promising characteristics of repeatability at same locations and separability between different locations. The main advantage of the signature based train localization method is the long-term stability without the use of absolute geographic position measurements of a GNSS. The long-term stability is achieved from a non-integrative signature match method. Furthermore, this signature method can cope with an along-track uncertainty of an estimator. The deviation of the along-track prediction can be observed with a shifted signature.

The reliability and accuracy of future train localization solutions can benefit from the proposed redundancy, especially in tunnels or may cope with changes of a signature. Furthermore, signature based train localization is considered as a valuable localization method for metro and subways.

\section{REFERENCES}

[1] J. Marais, J. Beugin, and M. Berbineau, "A survey of GNSS-based research and developments for the European railway signaling," IEEE Transactions on Intelligent Transportation Systems, 2017.

[2] E. Hedberg and M. Hammar, “Train localization and speed estimation using on-board inertial and magnetic sensors,” Master's thesis, Linköping University, Sweden., 2015.

[3] D. Titterton and J. Weston, Strapdown Inertial Navigation Technology, 2nd Edition. The Institution of Electrical Engineers (IEE), 2004.

[4] O. Heirich, B. Siebler, and E. Hedberg, "Study of train-side passive magnetic measurements with applications to train localizaiton," Hindawi Journal of Sensors, 2017

[5] B. Siebler, O. Heirich, and S. Sand, "Bounding INS positioning errors with magnetic-field-signatures in railway environments," in ION GNSS+ (to be published), 2017.

[6] O. Heirich, A. Steingass, A. Lehner, and T. Strang, "Velocity and location information from onboard vibration measurements of rail vehicles," in Information Fusion (FUSION), 2013 16th International Conference on, July 2013.

[7] O. Heirich and B. Siebler, "Train-side passive magnetic measurements," in IEEE International Instrumentation and Measurement Technology Conference (I2MTC), Pisa, Italy, May 2015.

[8] O. Heirich, P. Robertson, and T. Strang, "RailSLAM - localization of rail vehicles and mapping of geometric railway tracks," in International Conference on Robotics and Automation, IEEE ICRA, Mai 2013. 